\title{
Recent Developments and Validations in Geant4 Hadronic Physics
}

\author{
Dennis H. Wright, Tatsumi Koi*, Gunter Folger, Vladimir Ivantchenko, \\ Mikhail Kossov, Nikolai Starkov ${ }^{\dagger}$, Aatos Heikkinen** and \\ Hans-Peter Wellisch \\ ${ }^{*}$ Stanford Linear Accelerator Center, Menlo Park, California, USA \\ ${ }^{\dagger}$ CERN, Geneva, Switzerland \\ ${ }^{* *}$ Helsinki Institute of Physics, Helsinki, Finland \\ ${ }^{*}$ Geneva, Switzerland
}

\begin{abstract}
.
The Geant 4 hadronic models cover the entire range of energies required by calorimeters in new and planned experiments. The extension and improvement of the elastic, cascade, parameterized and quark-gluon string models will be discussed. Such improvements include the extension to more particle types, a review and correction of cross sections, and a better treatment of energy and momentum conservation. Concurrent with this development has been a validation program which includes comparisons with double differential cross sections. An ongoing hadronic shower validation will also be discussed which includes the examination of longitudinal shower shapes and the performance of the above models as well as their interaction with electromagnetic processes such as multiple scattering.
\end{abstract}

Keywords: simulation, Geant4, validation

PACS: 24.10.Lx, 02.70.Uu, 01.30.Cc

\section{INTRODUCTION}

For a given calorimeter simulation using Geant 4 [1, 2], a number of physical processes is assigned to each particle type. To each of these processes several models and cross section data sets are assigned. For the hadronic processes, the user must choose the models which are most appropriate to the energy range and level of detail required in his simulation. For calorimetry in LHC and ILC detectors, hadronic physics requirments are similar, so that a common set of processes and models may be used. These have been included in a few "physics lists" which users may invoke in their applications. Validation and subsequent improvement of these physics lists and their constituent processes and models is currently underway.

Elastic scattering, cascade, high energy and parameterized models will be discussed in particular, followed by selected highlights of the test-beam validation of showers.

\section{IMPROVEMENTS IN ELASTIC SCATTERING}

Elastic hadron scattering is important in the simulation of shower shapes and calorimeter response. The current elastic scattering model in Geant4 is derived from the GHEISHA 
code of Geant3 [3]. It does a reasonable job of reproducing the scattered angular distributions for angles smaller than about $15^{\circ}$. Beyond that it tends to over-estimate the large angle scattering. A better model has been developed which is relativistically correct, includes coherence effects such as diffraction minima due to the nuclear radius, and removes charge exchange as a separate reaction. In also includes improved $\mathrm{p}$ and $\mathrm{n}$ scattering from $\mathrm{p}, \mathrm{d}$, and He targets, as well as high precision neutron elastic scattering from isotopes.

\section{IMPROVEMENTS IN THE GEANT4 PARAMETERIZED MODEL}

Like the elastic scattering model, the Geant 4 parameterized models are derived from the GHEISHA models of Geant3. They are based on fits to experimental data over a broad energy range and employ theoretical guidance when little data is available. They are computationally fast, and may be used for all long-lived hadrons, at all incident energies.

This versatility is balanced by the approximate nature of these models. Originally formulated as a shower code, they do not conserve energy, momentum, charge or other quantum numbers on an event-by-event basis, but instead seek to conserve these quantities on average.

The approach of Geant 4 hadronics is to use these models only where speed is important, or when no better models exist. Current physics lists depend on the parameterized models over the incident particle energy range from $\sim 5 \mathrm{GeV}$ to $\sim 20 \mathrm{GeV}$, and at all energies for anti-baryons. Because of this dependence, and because many calorimeter studies employ energy flow methods, these models were recently improved with respect to energy conservation. An example of this improvement is shown in Fig. 1. The plot shows the total energy recovered from a $50 \mathrm{MeV}$ anti-neutron coming to rest in a perfect calorimeter. The sum of all the annihilation energies and masses should be $1.93 \mathrm{GeV}$. Using the original version of the model, the recovered energy has its mean at around $1.9 \mathrm{GeV}$, but the broad tails above and below the mean are problematic for any serious energy accounting. The new model enforces the $1.93 \mathrm{GeV}$ upper limit and narrows the energy distribution considerably.

Charge and baryon number conservation was also improved in the new version of these models, especially for hadron collisions with light nuclei.

\section{THE CASCADE MODELS}

In Geant 4 the energy range from 0 to $5-10 \mathrm{GeV}$ is handled by the so-called binary and Bertini cascade models. Both of these models are more detailed, and depend more heavily on theoretical arguments, than the parameterized models discussed previously. They also implicitly conserve energy-momentum and quantum numbers, and thus are good candidates to replace the parameterized models in this energy range.

The binary cascade model is valid for incident $p, n, \pi^{+}, \pi^{-}$from 0 to $3 \mathrm{GeV}$. It is based on hadron collisions with nucleons which form resonances. The resonances then decay according to their quantum numbers. This model was recently extended to include 


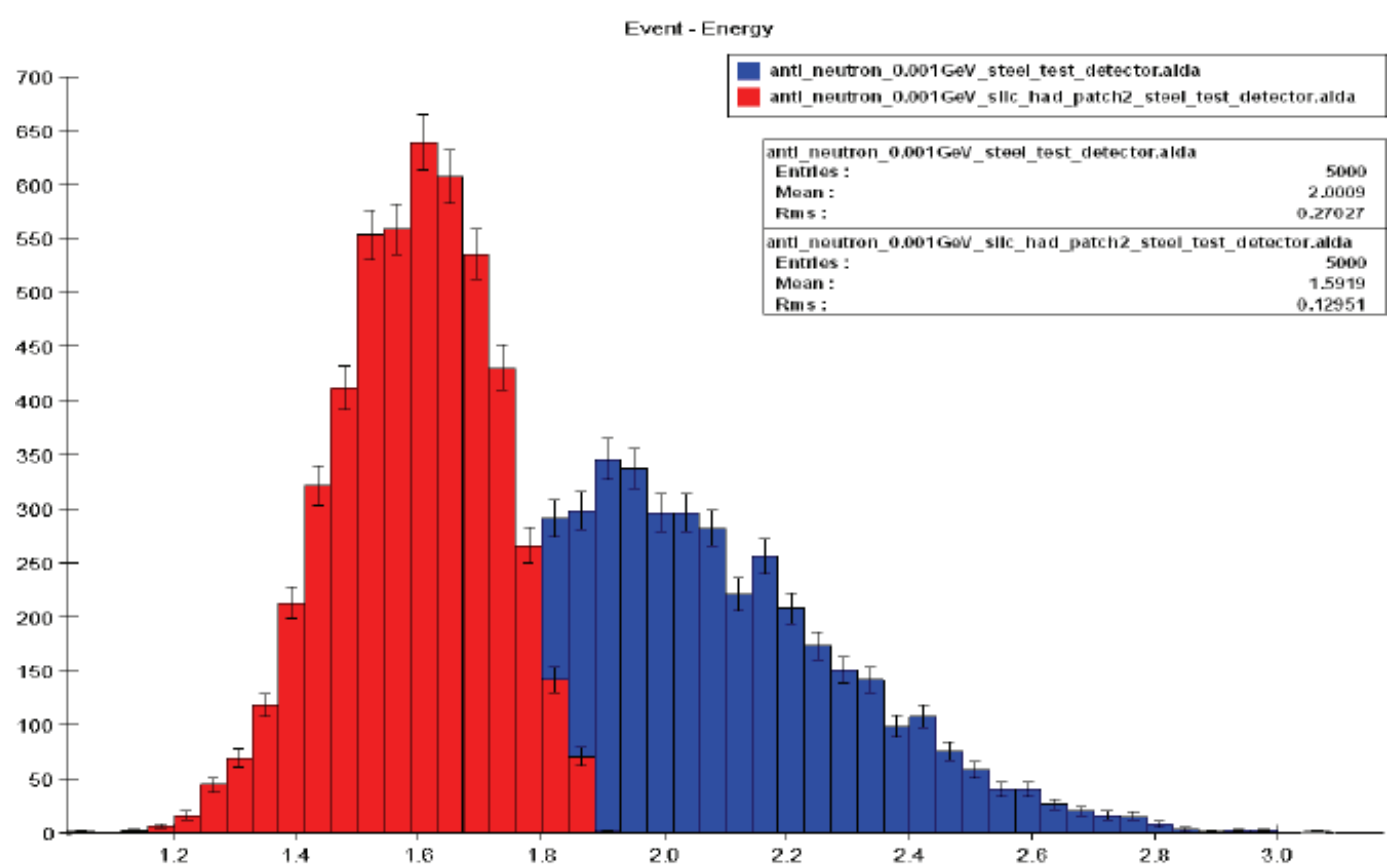

FIGURE 1. Recovered energy due to an annihilating anti-neutron in a perfect calorimeter. The dark gray histogram is the result of the original parameterized model and the light gray histogram is the result of the improved parameterized model. The horizonal axis is in GeV. Plot courtesy of Ron Cassell (SLAC).

ion-ion collisions, with the restriction that either the projectile or the target must have $A \leq 12$.

The Bertini cascade is based on the Bertini INC [4] code and is valid for $p, n, \pi^{+}, \pi^{-}$ from 0 to $10 \mathrm{GeV}$. Hadron collisions with nucleons are assumed to proceed according to the free-space partial cross sections and final state distributions measured for the incident particle types. This model was recently upgraded to handle elastic scattering, and the inelastic scattering of incident kaons and hyperons.

Both the Bertini and binary cascade model the target nucleus in detail, taking into account density variations and tracking in the nuclear field.

Both models have undergone extensive validation over the energy range $100 \mathrm{MeV}$ to $1 \mathrm{GeV}$. One such validation for incident $800 \mathrm{MeV}$ protons is shown in Fig 2. The binary cascade does a better job at reproducing the $7.5^{\circ}$ data, while the Bertini cascade does better at larger angles.

\section{THE HIGH ENERGY MODELS}

For incident particles of energy $15 \mathrm{GeV}$ to $\sim 10 \mathrm{TeV}$, three models are available in Geant4. The high energy parameterized (HEP) model is derived from the high energy part of GHEISHA, which was discussed previously. The quark gluon string (QGS) model is almost entirely a theory-based model which contains diffractive string excitation and string decay into hadrons. The Fritiof fragmentation (FTF) model is an alternate 


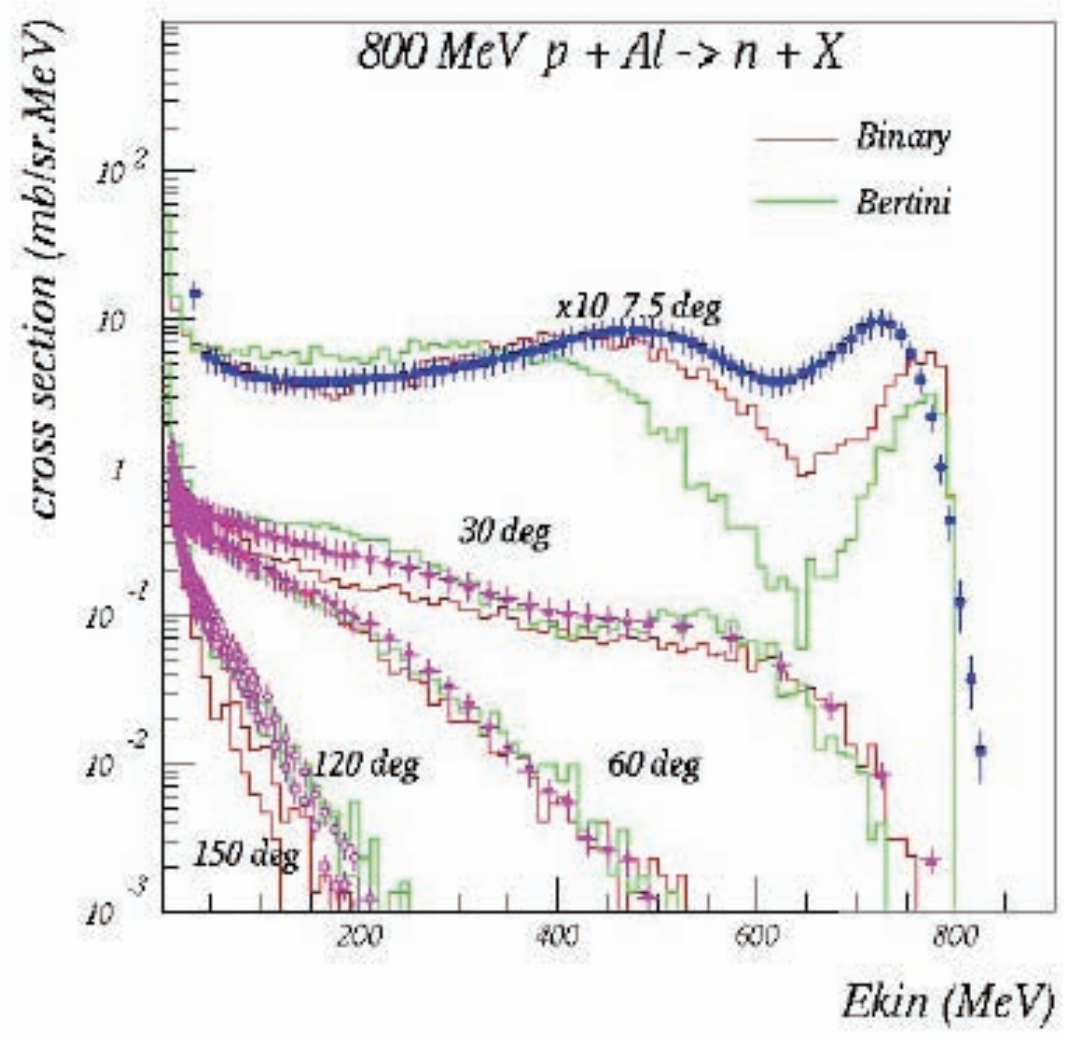

FIGURE 2. Neutron production from $800 \mathrm{MeV}$ protons on $\mathrm{Al}$. The double differential cross section $(\mathrm{mb} / \mathrm{sr} / \mathrm{MeV})$ vs. neutron kinetic energy $(\mathrm{MeV})$ is shown at $7.5,30,60,120$, and $150^{\circ}$. The dark gray histogram represents the Binary cascade and the light gray histogram represents the Bertini cascade. The data are from Ref. [5].

theoretical model with different string fragmentation functions. Of the two theoretical models (QGS and FTF), QGS works better in most cases. Most high energy calorimetry physics lists use either QGS or HEP models.

Validation of these models is underway. An example of the comparison of the QGS and HEP models is shown in Fig. 3. Here the $\pi^{+}$rapidity is examined for $320 \mathrm{GeV} / \mathrm{c}$ incident $\pi^{-}$. There are clear differences between the models: QGS produces a smooth curve with a central plateau, while HEP produces a three-peak structure. Both models agree reasonably well with the data, but one cannot currently be preferred over the other.

\section{SHOWER SHAPE STUDIES}

As mentioned in the introduction, a realistic Geant 4 simulation is built by combining physics processes and models into a physics list. Two such physics lists, named LHEP and QGSP, are the most used and most tested in high energy calorimetry. Shower shapes provide especially good integration tests of these physics lists and data from several 


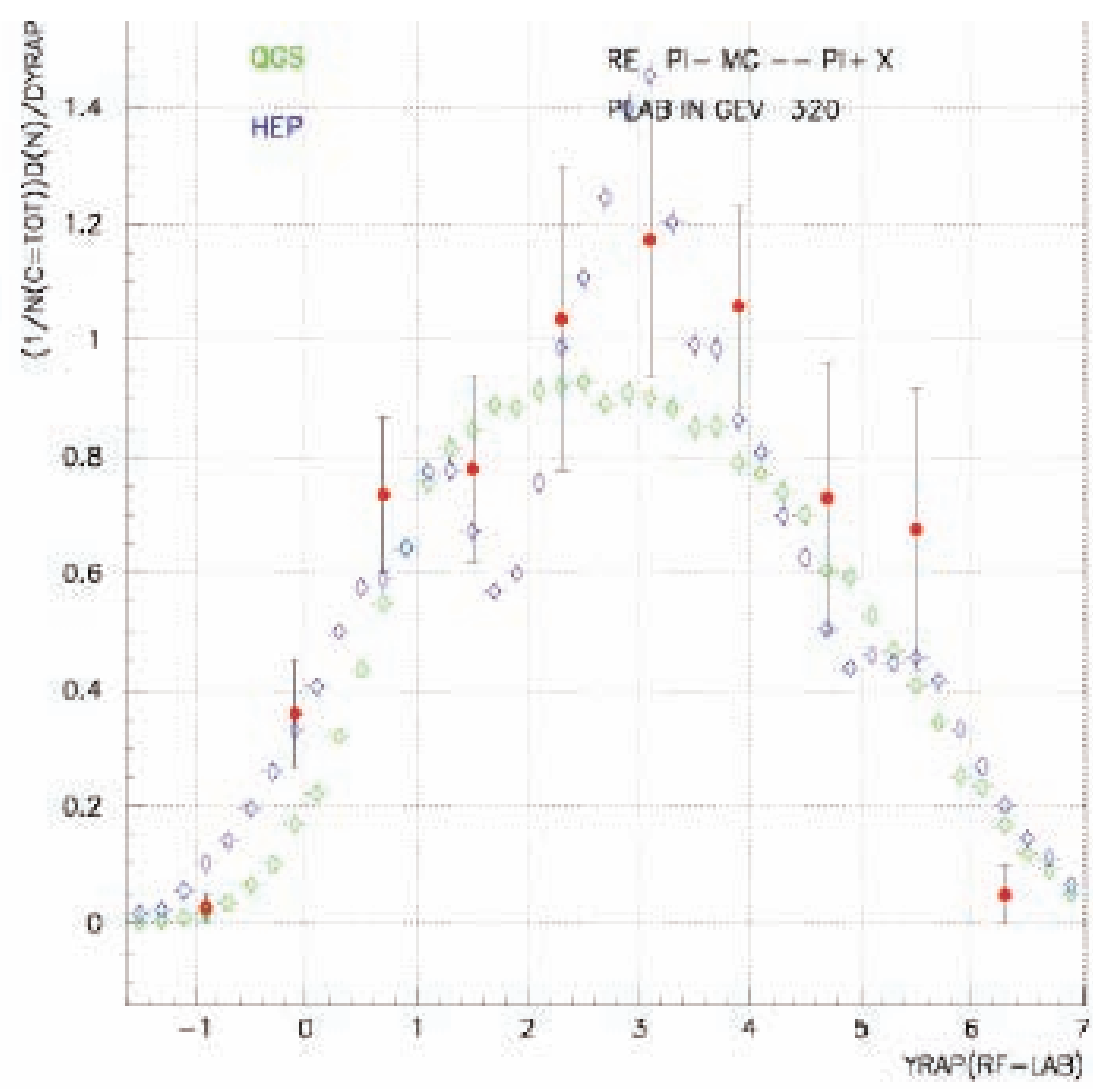

FIGURE 3. $\pi^{+}$yield as a function of rapidity for $320 \mathrm{GeV} / \mathrm{c} \pi^{-}$incident upon $\mathrm{Mg}$. Open diamonds represent the HEP prediction and open circles represent the QGS prediction. The data are from Ref. [6].

test-beam experiments have been compared to their predictions.

The LHEP physics list consists of the low energy parameterized (LEP) models and the high energy parameterized (HEP) models, plus the Geant4 standard electromagnetic package. The QGSP physics list consists of the quark gluon string (QGS) model, the precompound nucleus model, some of the LEP models, and the standard electromagnetic package.

An example of the shower shape studies using these physics lists is shown in Fig. 4. The data are from the CMS test-beam setup of 2004, which consisted of a $7 \times 7$ array of $\mathrm{PbWO}_{4}$ crystals and two wedges of alternating brass absorber and scintillator. Pion beams of between 2 and $300 \mathrm{GeV}$ were incident upon the detector. Longitudinal shower shapes, pion energy spectra and recovered energy were examined. From the longitudinal shower shape it is seen that the LHEP physics list does a good job of reproducing the profile. The QGSP physics list, however, produces a significantly shallower profile. The reasons for this are currently under study.

For lower beam energies, such as 5, 10 and $30 \mathrm{GeV}$, both QGSP and LHEP physics lists show good agreement with the shower shape data.

Pion energy spectra measured in the calorimeter are in good agreement with both models at all energies. 


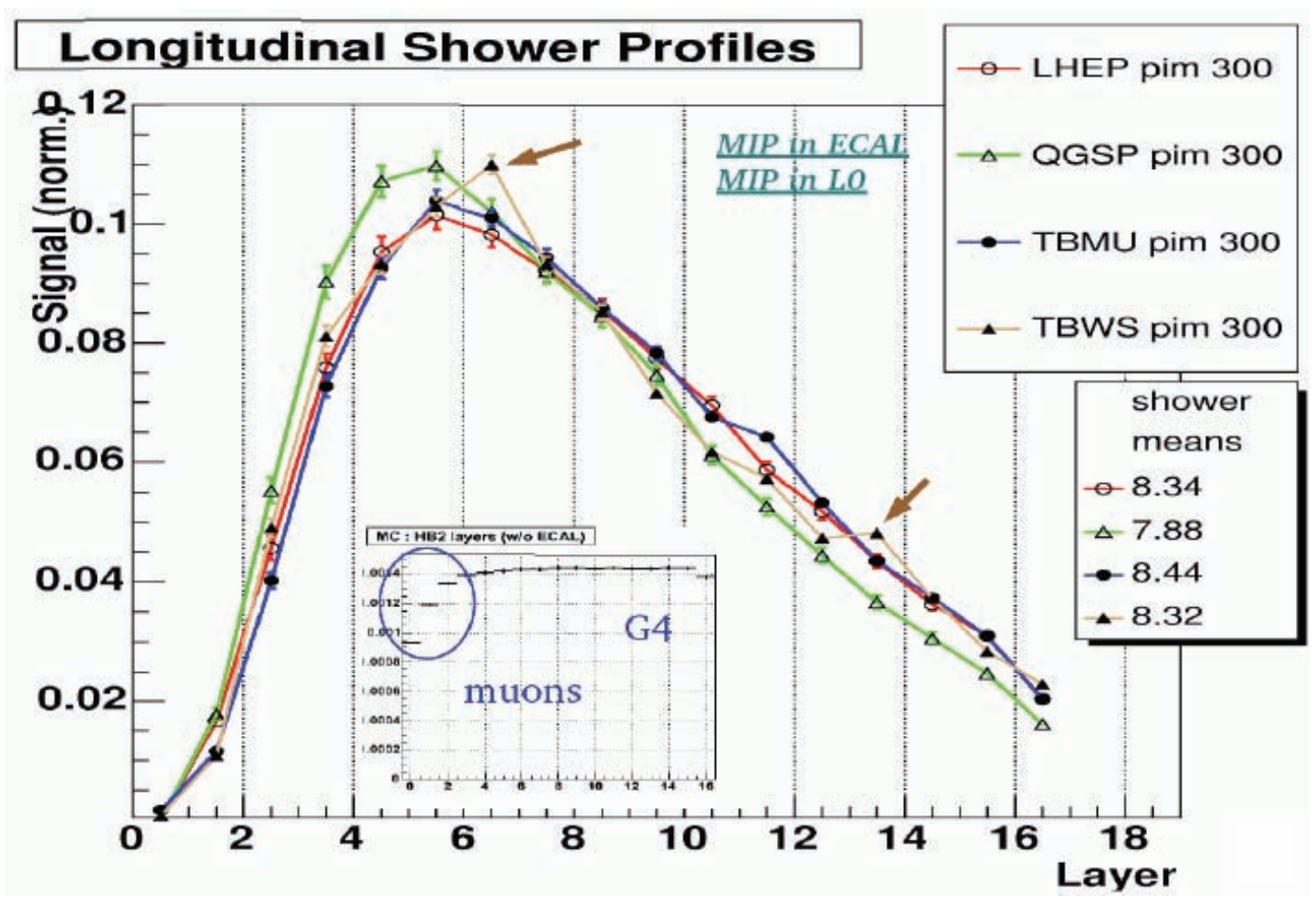

FIGURE 4. Longitudinal shower profile in the CMS test-beam calorimeter. Data are shown as filled triangles and filled circles connected by lines to guide the eye. Predictions of the LHEP physics list are shown by open circles and predictions of the QGSP physics list are shown by open triangles. The vertical axis is the normalized energy deposit and the horizontal axis is the hadronic calorimeter layer in which the energy is depositied. Plot courtesy of J. Damgov and the CMS collaboration.

In other measures, such as recovered energy, again the agreement with both physics lists is good, as shown in Fig. 5.

\section{OTHER DEVELOPMENTS AND VALIDATIONS}

Models dealing with the energy range 1-15 GeV have until recently been difficult to validate due to a scarcity of data. A number of test-beam experiments have been performed which now provide more data in this range. For example, the HARP experiment has recently published data for $12.9 \mathrm{GeV} / \mathrm{c}$ incident hadrons [7]. This will be used to validate and tune the LEP models in Geant4.

Shower shapes and calorimeter response are certainly dependent on the interaction cross sections. A review of all hadronic cross sections in Geant4 is now underway. Already the cross sections internal to the QGS model have been improved. This may lead to improved shower shapes at high energies. 


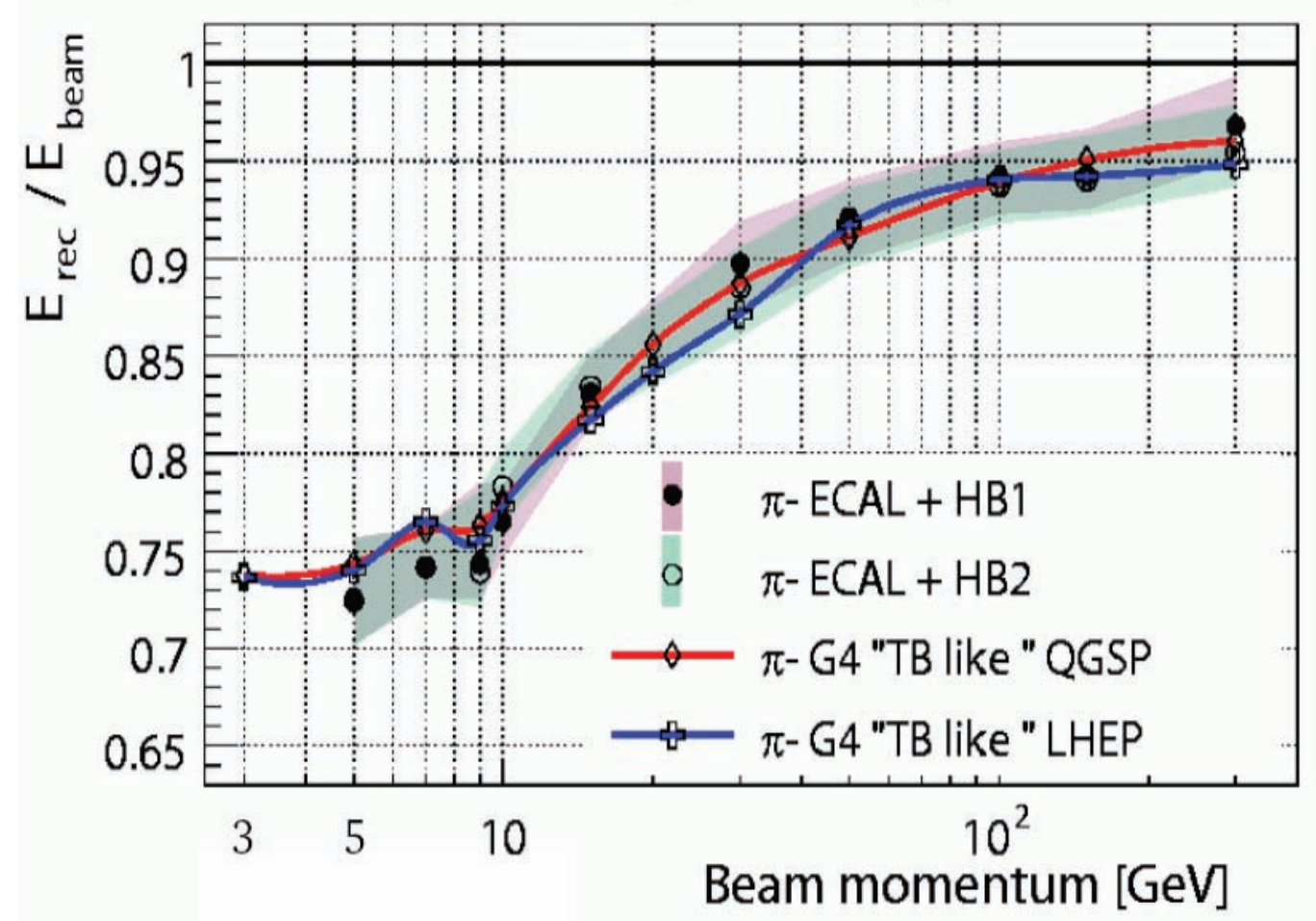

FIGURE 5. Recovered energy in CMS test-beam calorimeter as a function of incident particle momen$\operatorname{tum}(\mathrm{GeV} / \mathrm{c})$. Data are shown as filled and open circles (with error bands). Predictions of the LHEP physics list are shown as open crosses and predictions of the QGSP physics list are shown as open diamonds. Plot courtesy of J. Damgov and the CMS collaboration.

\section{CONCLUSION}

Many improvements to the Geant4 hadronic models are being made in order to improve calorimeter response and shower shape agreement. Elastic scattering is important in shower development and new models are now available which are more physical. The low energy and high energy parameterized models of Geant 4 are still widely used and improvements have been made to them with respect to energy conservation. However, new, more physical models are being substituted where appropriate. The binary and Bertini cascade models are being used and improved as is the quark gluon string model. These models have been combined into the LHEP and QGSP physics lists, which are in the process of being validated against test-beam data from various high energy calorimeters. Agreement with CMS test-beam data is good for the most part, but some deviations of the QGSP list are being examined.

\section{ACKNOWLEDGMENTS}

This work was supported by the Stanford Linear Accelerator Center (SLAC) through a grant from the U.S. Department of Energy. It was also supported by the European 
Organization for Nuclear Research (CERN) and the Helsinki Institute of Physics.

\section{REFERENCES}

1. S. Agostinelli et al., Nucl. Instr. Meth. Phys. Res. A 506 (2003) 250-303.

2. J. Allison et al., IEEE Trans. Nucl. Sci. 53 No. 1 (2006) 270-278.

3. GEANT - Detector description and simulation tool, CERN Program Library Long Write-up W5013, CERN Geneva.

4. H.W. Bertini and P. Guthrie, Nucl. Phys. A169 (1971).

5. W.B. Amian et al., Nucl. Sci. and Eng. 112 (1992) 78.

6. J.J. Whitmore et al., Z.Phys. C62 (1994) 199.

7. The HARP collaboration, Nucl. Phys. B732 (2006) 1. 